\title{
Artificial Intelligence Framework for Efficient Detection and Classification of Pneumonia Using Chest Radiography Images
}

\author{
Ali Mohammad Alqudah ( $\boldsymbol{D}$ ali_qudah@hotmail.com ) \\ Yarmouk University https://orcid.org/0000-0002-5417-0043 \\ Shoroq Qazan \\ Yarmouk University \\ Ihssan S. Masad \\ Yarmouk University
}

\section{Research}

Keywords: Artificial Intelligence, Deep Learning, Machine Learning, Framework, Pneumonia, x-ray

Posted Date: March 18th, 2021

DOI: https://doi.org/10.21203/rs.3.rs-66836/v2

License: (1) (i) This work is licensed under a Creative Commons Attribution 4.0 International License.

Read Full License

Version of Record: A version of this preprint was published at Journal of Medical and Biological Engineering on June 14th, 2021. See the published version at https://doi.org/10.1007/s40846-02100631-1. 


\section{Abstract}

\section{Background}

Chest diseases are serious health problems that threaten the lives of people. The early and accurate diagnosis of such diseases is very crucial in the success of their treatment and cure. Pneumonia is one of the most widely occurred chest diseases responsible for a high percentage of deaths especially among children. So, detection and classification of pneumonia using the non-invasive chest $x$-ray imaging would have a great advantage of reducing the mortality rates.

\section{Results}

The results showed that the best input image size in this framework was 6464 based on comparison between different sizes. Using $\mathrm{CNN}$ as a deep features extractor and utilizing the 10-fold methodology the propose artificial intelligence framework achieved an accuracy of $94 \%$ for SVM and $93.9 \%$ for $\mathrm{KNN}$, a sensitivity of $93.33 \%$ for SVM and $93.19 \%$ for KNN and a specificity of $96.68 \%$ for SVM and $96.60 \%$ for KNN.

\section{Conclusions}

In this study, an artificial intelligence framework has been proposed for the detection and classification of pneumonia based on chest $x$-ray imaging with different sizes of input images. The proposed methodology used CNN for features extraction that were fed to two different types of classifiers, namely, SVM and KNN; in addition to the SoftMax classifier which is the default CNN classifier. The proposed $\mathrm{CNN}$ has been trained, validated, and tested using a large dataset of chest $\mathrm{x}$-ray images contains in total 5852 images.

\section{Background}

Many terms such as peripleumoniacon, pleurisy, and peripneumony were used by ancient Romans and Greeks to describe an illness which includes many conditions that are currently known as pneumonia;

and later in the $19^{\text {th }}$ century, a scientist called Laennec distinguished 'pleurisy' from pneumonia, and after that another scientist named Rokitansky recognized the bronchopneumonia and lobar pneumonia as different pathological entities [1, 2]. Nowadays, in the US only as an example, there are more than 1 million pneumonia patients that are hospitalized with approximately 50,000 deaths. Globally, around 450 million people get infected with pneumonia per year, amongst which 4 million people die [2]. Currently, the best available, and most commonly used, technology to diagnose pneumonia is Chest x-ray imaging which plays a vital and crucial role in the daily clinical care of pneumonia patients [2, 3].

The development and evolution of computerized technologies especially Al with its different branches have made the detection and diagnosis of diseases more accurate and made medical decisions more precise and effective $[4,5]$. The detection and classification of pneumonia has gained the attention of 
researchers who have provided different types of $M L$ and $D L$ techniques for pneumonia detection with acceptable accuracy $[3,5,6]$. On the other hand, one of the most challenging tasks in detecting pneumonia using chest $x$-ray images is that this process completely depends on the availability of expert radiologists who have the ability to correctly detect pneumonia and requires laboratory tests to differentiate its viral type from bacterial type $[3,6,7]$. During traditional detection of pneumonia, expert radiologists search for white spots on the chest $x$-ray image that indicate an infection; in addition to the bright areas that represent pneumonia fluids in the lungs [8, 9]. However, because of the limited color distribution of x-ray images (Gray Levels), it will consist of shades of black and white which makes it a challenge to decide whether there is a pneumonia infection in the lungs or not [6,9]. Figure 1 shows Chest $x$-ray images with normal, viral pneumonia, and bacterial pneumonia conditions.

In the current research work, a hybrid artificial intelligence methodology that combines deep learning and machine learning techniques has been proposed. In this methodology, a CNN has been applied and used for feature extraction instead of conventional methods to detect and classify pneumonia, the CNN was applied using different input image sizes to find the optimal size, and then these extracted features were fed into two different machine learning algorithms (SVM and KNN); in addition to the softmax classifier which is the default classifier for CNN. In general, the original contributions of this work include proposing a new CNN architecture to detect pneumonia from Chest x-ray images rather than AlexNet, GoogleNet, ResNet, and DenseNet; as well as using the proposed architecture to extract automated deep features and pass them to machine learning algorithms to detect and classify the pneumonia.

\section{Literature Review}

Deniz et. al. [10] provided a deep learning-based method for pneumonia detection from chest x-ray images. They proposed a methodology with modified contrast images to increase the accuracy of the proposed model. They used digital image processing techniques that were able to enhance the ROI in which the ResNet based CNN model focus during the training process, the authors reported an accuracy of $78.73 \%$. On the other hand, Okeke et. al. [11] proposed another deep learning approach which was efficient in pneumonia classification. The authors proposed a new dense $\mathrm{CNN}$ architecture designed by them that consisted of 8 layers only, the authors provided a comparison between different input image sizes and found that the best input size was $200 \times 200 \times 3$ which scored a validation accuracy of $93.73 \%$.

Benjamin et. al. [12] proposed a supervised learning technique for pneumonia detection. The technique was based on deep learning methods using DenseNet based architecture which was trained using the adaptive method on 5606 random images with a size of $32 \times 32$ because of memory limitation. The authors reported an AUC of 0.609 for the proposed network. Saraiva et. al. [13] proposed a neural network-based methodology for pneumonia detection using both MLP and CNN. The authors used the KFold cross-validation for training the models and achieved an accuracy of $94.40 \%$ and $92.16 \%$ for CNN and MLP, respectively. 
Gu et. al. [14] proposed a methodology for the classification of pneumonia chest x-ray images from three datasets: JSRT, MC, and Guangzhou Women and Children's Medical Center with total number of 4892 images. The authors at first segmented the region of interests (ROI) which was the right and left lungs using FCN model using pre-trained AlexNet model. After the segmentation of the lungs, different types of features were extracted including wavelet features, handcraft features, HOG features, GLCM features, and DCNN features. Finally, these features were fed separately to SVM, and the best result was obtained when using DCNN features with an overall accuracy of $80.48 \%$.

Rahman et. al. [15] proposed a transferee learning-based methodology for classification and detection of pneumonia (both viral and bacterial). The authors proposed a comparison study between different predesigned CNN architectures to find the best one that achieved the highest performance. The results showed that DenseNet201 had the best performance among all used architectures where it scored an accuracy of $98 \%$ for normal vs pneumonia and $93.3 \%$ for normal vs viral pneumonia vs bacterial pneumonia; while the accuracy was $95 \%$ for viral pneumonia vs bacterial pneumonia.

On the other hand, Rajaraman et. al. [16] proposed another transferee learning-based methodology for classification and detection of viral and bacterial pneumonia. The methodology was tested among two datasets baseline and cropped ROI using four different architectures (customized VGG16, sequential, inception, and residual). The customized VGG16 outperformed all other architectures and achieved an accuracy of $95.7 \%, 93.6 \%$, and $91.7 \%$ for normal vs pneumonia, normal vs viral pneumonia vs bacterial pneumonia, and viral pneumonia vs bacterial pneumonia, respectively, using baseline dataset. While it achieved an accuracy of $96.2 \%, 93.6 \%$, and $91.8 \%$ for normal vs pneumonia, normal vs viral pneumonia vs bacterial pneumonia, and viral pneumonia vs bacterial pneumonia, respectively, using cropped ROI dataset.

\section{Results And Discussion}

The hardware environment for testing the proposed algorithm was a desktop computer with Intel Core 176700 at $3.4 \mathrm{GHz}$ and $16 \mathrm{~GB}$ of RAM and the code has been executed using a parallel environment. At first, the system has been tested for different input image sizes to select the best size that is able to extract the best features to distinguish between the three classes. In this stage, the data was separated into two subsets; $70 \%$ of data was used for training and the other $30 \%$ was for validation to feed all CNNs. Figure 4 shows the training accuracy and loss for different input image sizes, while Table 3 shows the performance evaluation of the four different input sizes.

Table 3 Validation Performance Evaluation for Different Input Image Sizes.

\begin{tabular}{ccccc}
\hline Input Size & Accuracy (\%) & Sensitivity (\%) & Specificity (\%) & Precision (\%) \\
\hline $32 \times 32$ & 76.99 & 75.17 & 88.00 & 76.29 \\
$64 \times 64$ & 80.07 & 79.24 & 89.55 & 78.80 \\
\hline $128 \times 128$ & 79.50 & 78.86 & 89.23 & 78.14 \\
\hline $256 \times 256$ & 78.59 & 77.64 & 88.86 & 76.88 \\
\hline
\end{tabular}


Figure 5 shows the class activation maps for different cases using the last ReLU layer of the used CNN architecture. The CAM shows that the system has selected the proper ROI to distinguish between the three cases correctly.

Based on the results obtained and shown in Table 3, the best image input size was $64 \times 64$; and so, this input size was chosen for further processes and for feature extraction from FC layer. Figure 6 (A) shows the extracted features space from the whole dataset for two classes (Normal and Pneumonia). While Figure 6 (B) shows the extracted features space from the whole dataset for the three classes (Normal, Bacterial Pneumonia, and Viral Pneumonia).

As shown in Figure 6 A, Pneumonia can be detected easily and accurately using these features because the classes are well separated. However, dividing pneumonia into bacterial and viral types make it more difficult to classify them with a high accuracy because of their overlapped features as shown in Figure 6 B. This overlap of features spaces requires a classifier that is able to discriminate the classes effectively which cannot be accomplished by SoftMax classifier. So, after the feature extraction was executed, the extracted features were fed to two different types of classifiers (KNN and SVM). Because there was an overlap between the bacterial and viral features, the classifiers have been trained using $10 \mathrm{~K}$-Fold CrossValidation methodology.

K-Fold Cross-Validation methodology was used to make a generalization for classifiers models and make a perfect fitting on data by dividing the data (features space) into K groups, and Cross-Validation ensured that each fold from the K-Folds has been used as a testing set. The KNN and SVM classifiers have been trained using $10 \mathrm{~K}$-Fold Cross-Validation and the obtained models have been saved for further results analysis. Figure $7 \mathrm{~A}$ and $\mathrm{B}$ show the Confusion Matrix for the KNN classifier and the ROC of the same classifier, while Figure $8 \mathrm{~A}$ and $\mathrm{B}$ show the Confusion Matrix for the SVM classifier and the ROC of the same classifier.

For further analysis of the extracted features and the used classifiers, more performance evaluation metrics (Sensitivity, Specificity, and Precision) have been calculated using the generated confusion matrices. Based on these values, the used classifiers provided outperforming results and were able to discriminate between the normal and pneumonia and between the two types of pneumonia: the bacterial and viral types. Table 4 shows the performance evaluation of the used two classifiers to distinguish between the three classes (Normal vs Bacterial Pneumonia vs Viral Pneumonia) where SVM classifier slightly outperformed the KNN classifier; i.e, the used classifiers were both suitable for the extracted features with a high efficiency of classification.

Table 4 Performance Evaluation for the Used Two Classifiers.

\begin{tabular}{ccccc}
\hline Method & Accuracy (\%) & Sensitivity (\%) & Specificity (\%) & Precision (\%) \\
\hline CNN-KNN & 93.89 & 93.19 & 96.60 & 94.10 \\
\hline CNN-SVM & 94.03 & 93.33 & 96.68 & 94.22 \\
\hline
\end{tabular}

As an extended analysis for the classifiers, performance evaluation measures (Accuracy, Sensitivity, Specificity, and Precision) have been calculated for each single data class. Table 5 shows the 
performance evaluation of the three classes for each classifier. It is shown that the lowest performance of the system was for classifying viral pneumonia, while the highest was for the Normal class. In general, as noticed from Figures (7 and 8 ) and Tables 4 and 5, the used methodology and classifiers have a high performance for detection and classification of Pneumonia from Chest x-ray images, and it outperforms the CNN using Softmax classifier which had a maximum accuracy of $80.07 \%$ as seen in Table 3 . The performance evaluation values of both Bacterial Pneumonia and Viral Pneumonia were lower than the Normal class because it is a difficult task to detect and classify pneumonia solely from chest x-ray images, in addition to the required knowledge of disease pathology and human anatomy.

Table 5 Performance Evaluation of Each Data Class for the Used Two Classifiers.

\begin{tabular}{ccccccc}
\hline Method & \multicolumn{3}{c}{ CNN-KNN } & \multicolumn{3}{c}{ CNN-SVM } \\
\hline Class & Normal & $\begin{array}{c}\text { Pneumonia } \\
\text { Bacterial }\end{array}$ & $\begin{array}{c}\text { Pneumonia } \\
\text { Viral }\end{array}$ & Normal & $\begin{array}{c}\text { Pneumonia } \\
\text { Bacterial }\end{array}$ & $\begin{array}{c}\text { Pneumonia } \\
\text { Viral }\end{array}$ \\
\hline Accuracy \% & 98.42 & 96.08 & 85.06 & 98.32 & 96.18 & 85.60 \\
Sensitivity \% & 98.40 & 96.1 & 85.12 & 98.35 & 96.21 & 85.64 \\
\hline Specificity \% & 99.16 & 93.27 & 97.38 & 99.20 & 93.46 & 97.38 \\
\hline Precision \% & 97.74 & 92.80 & 91.76 & 97.86 & 93.00 & 91.81 \\
\hline
\end{tabular}

\section{Conclusion And Future Work}

In this research study, an original new artificial intelligence system has been proposed and evaluated for the detection and classification of bacterial Pneumonia and viral Pneumonia as well as normal cases using chest $x$-ray images. The hybrid artificial intelligence model has been built using a CNN model which pretrained on other medical images (OCT images), the proposed methodology was different from the other methods in the literature that depended heavily on the transfer learning approach using a pretrained CNN architecture and modified version of them only. The results of the current study indicated that the proposed hybrid system outperformed previous systems and it was able to detect and classify Pneumonia efficiently (accuracy of $94 \%$ ) from chest x-ray images. In future, this work will be extended and enhanced to detect other pulmonary diseases using chest $x$-ray images.

\section{Materials And Methods}

In this section, the materials and methods used in this research paper will be discussed in detail. Figure 2 shows the block diagram of the proposed methodology.

\subsection{Dataset}

The original dataset which was published by kermany et. al. [17] is consisting of three main folders: the training, testing, and validation folders; and inside each folder there are two subfolders one of them contains pneumonia chest $x$-ray images while the other contains normal chest $x$-ray images. A total of 5,852 Chest $x$-ray images of anterior-posterior cross-section were carefully chosen from retrospective pediatric patients between 1 and 5 years old [15]. The entire pneumonia chest $x$-ray images were named 
with bacteria or virus and these labels were used to split the pneumonia folder into two subfolders: viral pneumonia and bacterial pneumonia. Because of the small size of validation and testing images, and in order to balance the proportion of data assigned to the entire dataset, the original data categories were modified and combined, then the entire data was rearranged into a training set, validation, and testing sets with portion of $70 \%, 15 \%$, and $15 \%$, respectively. A total of 4,097 images were allocated to the training set and 877 images were assigned to the validation sets to improve the validation accuracy and 878 images allocated for testing dataset for test the system during $\mathrm{K}$-fold process.

Table 1 The distribution of images used in the system.

\begin{tabular}{ccccc}
\hline Case & $\begin{array}{c}\text { Number of Training } \\
\text { Images }\end{array}$ & $\begin{array}{c}\text { Number of Validation } \\
\text { Images }\end{array}$ & $\begin{array}{c}\text { Number of Testing } \\
\text { Images }\end{array}$ & $\begin{array}{c}\text { Total Number of Total } \\
\text { Images }\end{array}$ \\
\hline Normal & 1,107 & 237 & 237 & 1,581 \\
$\begin{array}{c}\text { Pneumonia } \\
\text { Bacterial }\end{array}$ & 1,945 & 416 & 417 & 2,778 \\
\hline $\begin{array}{c}\text { Pneumonia } \\
\text { Viral }\end{array}$ & 1,045 & 224 & 224 & 1,493 \\
Total & $\mathbf{4 , 0 9 7}$ & $\mathbf{8 7 7}$ & $\mathbf{8 7 8}$ & $\mathbf{5 , 8 5 2}$ \\
\hline
\end{tabular}

\subsection{CNN Architecture}

The modified CNN model that has been used in this work was initially proposed by Alqudah [18]. Any CNN model consists of two major stages: the feature extractions stage and the classification stage. Each stage contains a set of layers; the feature extraction layers immediately take the previous layer's output as input, and its output is passed to the next layer as an input. While the classification stage layers are placed at the end of the CNN model $[9,10,18]$. The classifier layer requires individual features (vectors) as input to perform computations like any classifier. Figure 3 shows the modified model that has been used, while Table 2 shows the details of the modified CNN model.

Table 2 Layers Information for Proposed CNN Architecture. 


\begin{tabular}{|c|c|c|c|c|c|c|c|}
\hline \# & Layer & Information & & \# & Layer & Information & \\
\hline \multirow[t]{2}{*}{1} & Input Layer & Size & $64 * 64$ & 9 & Maxpol_2 & Kernel Size & $2 * 2$ \\
\hline & & & & & & Stride & $2 * 2$ \\
\hline 2 & Conv_1 & Number of Filters & 32 & 10 & Conv_3 & Number of Filters & 32 \\
\hline & & Kernel Size & $3 * 3$ & & & Kernel Size & $3 * 3$ \\
\hline & & Activation & RELU & & & Activation & RELU \\
\hline 3 & Batch_Norm_1 & Number of Channels & 32 & 11 & Batch_Norm_3 & Number of Channels & 32 \\
\hline 5 & Maxpol_1 & Kernel Size & $2 * 2$ & 13 & Maxpol_3 & Kernel Size & $2 * 2$ \\
\hline & & Stride & $2 * 2$ & & & Stride & $2 * 2$ \\
\hline \multirow[t]{3}{*}{6} & Conv_2 & Number of Filters & 16 & 14 & Conv_4 & Number of Filters & 32 \\
\hline & & Kernel Size & $3 * 3$ & & & Kernel Size & $3 * 3$ \\
\hline & & Activation & RELU & & & Activation & RELU \\
\hline 7 & Batch_Norm_2 & Number of Channels & 16 & 15 & Batch_Norm_4 & Number of Channels & 32 \\
\hline
\end{tabular}

\subsection{Deep Feature Extraction using CNN}

The modified CNN model (AOCT-Net), initially proposed and designed by Alqudah [18], was retrained on Chest x-ray images dataset and then used for deep feature extraction process. This CNN model was designed for classification of OCT images into five different classes and used in this research as deep features extraction from chest $x$-ray images. In this paper, the FC is used as feature extraction layer, this layer precedes the classification layer (SoftMax Classifier); i.e., it will produce features vectors contain three features, each of which is used to describe one type of the classes $[19,20]$. Such feature extraction technique is very efficient and able to extract very deep and selective features that are very representative for the entered data especially when the used CNN is designed well [18]. The number of extracted features from this method is the same as the number of classes where each feature is responsible for representing a certain class. Features space extracted using such method consists of an array of features ( ) where represents number of entered data (Signals or Images) and is the number of classes [19].

\subsection{Class Activation Mapping (CAM)}

CAM is used to visualize the results of the use of CNN to localize the targeted image regions for feature extraction. The probability for each class of a single image predicted using the trained CNN for each class gets mapped back over the input image to the final convolutional layer of the respective network to highlight the discriminative regions that are specific to each class [20]. The CAM for a specific class will result from the activation map of the last ReLU (Rectified Linear Unit) layer of the CNN which usually precedes the fully connected layer or after the final convolutional layer. Using this method, we can determine how much each activation contributes to the final score of that particular class. Therefore, it allows distinguishing the areas within an image that differentiates the class specificity prior to the softmax layer, which leads to the probability predictions [20]. 


\subsection{Classification Stage}

After feature extraction, the classifier is needed to find the corresponding class for every input test image. In literature, different types of classification algorithms have been used to accomplish this task, such as Support SVM, KNN, and ANN. In this research paper, SVM and KNN have been trained using $10 \mathrm{~K}$-Fold techniques to generalize the classification model.

\subsubsection{Support Vector Machine (SVM) Classifier}

SVM is one of the known and most widely used supervised machine learning algorithms which is mainly used for classifying data into two main categories and later on has been expanded for multiclass classification [20]. During the training of SVM, it uses a specified training partition of the data to build a model that represents a hyperplane model used for expecting the new testing partition of the class. The main simple idea of the SVM is to find the best hyperplane that is able to separate the training dataset into two classes. This hyperplane will maximize the margin between the nearest data point and the hyperplane [21]. Since introducing SVM, it has been successfully applied to a wide range of medical applications including breast cancer diagnosis [21], melanoma skin cancer [23], and histopathological slices recognition [24].

\subsubsection{K-Nearest Neighbor (KNN) Classifier}

$\mathrm{KNN}$ is a well-known and widely used unsupervised machine learning algorithm which is mainly used for clustering the input data into main clusters (categories) [20]. KNN algorithm can be used for two main types of problem: classification and regression. KNN has different properties such as it is simple, lazy, non-parametric, and instant-based learning [25]. For this kind of problems, the input data vector must consist of the feature data (space) while the output data vector contains the class member that is obtained using the majority vote technique from it is neighbor's classes. The majority voting technique is applied to the weights representing the distance between each feature space point and the center of mass of the input data vector $[20,25]$.

\subsection{Performance Evaluation}

In any Al based system there is must be an evaluation of the system performance regarding any new data. To evaluate the performance of the proposed hybrid system, the original annotations of the x-ray chest images have been compared to the same images annotations generated by the system. Then based on these annotations, the accuracy, sensitivity, precision, and specificity have been calculated. These measures indicate how precisely the $x$-ray chest images are diagnosed [26]. To compute these measures, four different types of statistical values are computed which are TP, FP, FN and TN [27, 28]. Then using these values, the mentioned measurements have been computed as follows: 


$$
\begin{aligned}
& \text { Accuracy }=\frac{\mathrm{TP}+\mathrm{TN}}{\mathrm{TP}+\mathrm{FP}+\mathrm{TN}+\mathrm{FN}} \\
& \text { Sensitivity }=\frac{\mathrm{TP}}{\mathrm{TP}+\mathrm{FN}} \\
& \text { Specificity }=\frac{\mathrm{TN}}{\mathrm{FP}+\mathrm{TN}} \\
& \text { Precision }=\frac{\mathrm{TP}}{\mathrm{FP}+\mathrm{TP}}
\end{aligned}
$$

\section{Abbreviations}

CNN: Convolutional Neural Network; SVM: Support Vectors Machine; KNN: K-nearest neighbor with Euclidean distance equal weight; US: United States; Al: artificial intelligence; ML: Machine Learning; DL: Deep Learning; MLP: Multi-Layer Perceptron; JSRT: Japanese society of radiological technology; MC: Montgomery County, Md; FCN: fully convolutional networks; DCNN: Deep Convolutional Neural Network; ROI: Region of Interest; OCT: optical coherence tomography; FC: fully connected; CAM :Class Activation Mapping; ANN: Artificial Neural Network; TP: True positive; TN: True negative; FP: False positive; FN: False negative.

\section{Declarations}

\section{Acknowledgements}

We would like to acknowledge the Springer Nature for the waiving of the article processing charges.

\section{Authors' contributions}

A.M.Q and S.Q accomplished the machine learning models development. I.S.M accomplished the data and results analysis. All authors accomplished the manuscript writing and revising. All authors read and approved the final manuscript.

\section{Funding}

This work does not receive any funding or support.

\section{Availability of data and materials}

The dataset analyzed during the current study was derived from the following public domain resources: https://www.kaggle.com/paultimothymooney/chest-xray-pneumonia

\section{Consent for publication}

All authors consent for the publication of this manuscript.

\section{Competing interests}

The authors declare that they have no competing interests. 


\section{Ethics approval and consent to participate}

Not applicable.

\section{References}

1. Woodhead M. Pneumonia classification and healthcare-associated pneumonia: a new avenue or just a cul-de-sac? Thorax 2013;68:985-986. DOI: 10.1136/thoraxjnl-2013-204060.

2. Mackenzie G. The definition and classification of pneumonia. Pneumonia. 2016 Dec;8(1):14. DOI: 10.1186/s41479-016-0012-z.

3. Ching T, Himmelstein DS, Beaulieu-Jones BK, Kalinin AA, Do BT, Way GP, Ferrero E, Agapow PM, Zietz M, Hoffman MM, Xie W. Opportunities and obstacles for deep learning in biology and medicine. Journal of The Royal Society Interface. 2018 Apr 4;15(141):20170387. DOI: 10.1098/rsif.2017.0387.

4. Lakhani P, Sundaram B. Deep learning at chest radiography: automated classification of pulmonary tuberculosis by using convolutional neural networks. Radiology. 2017 Apr 24;284(2):574-82. DOI: 10.1148/radiol.2017162326.

5. Maier A, Syben C, Lasser T, Riess C. A gentle introduction to deep learning in medical image processing. Zeitschrift für Medizinische Physik. 2019 May 1;29(2):86-101. DOI:

10.1016/j.zemedi.2018.12.003.

6. Rajpurkar P, Irvin J, Zhu K, Yang B, Mehta H, Duan T, Ding D, Bagul A, Langlotz C, Shpanskaya K, Lungren MP. Chexnet: Radiologist-level pneumonia detection on chest x-rays with deep learning. arXiv preprint arXiv:1711.05225. 2017 Nov 14.

7. Mazurowski MA, Buda M, Saha A, Bashir MR. Deep learning in radiology: an overview of the concepts and a survey of the state of the art. arXiv preprint arXiv: 08717. 2018 Feb 10.

8. Mazurowski MA, Buda M, Saha A, Bashir MR. Deep learning in radiology: An overview of the concepts and a survey of the state of the art with focus on MRI. Journal of Magnetic Resonance Imaging. 2019 Apr;49(4):939-54. DOI:10.1002/jmri.26534.

9. Abiyev RH, Ma'aitah MK. Deep convolutional neural networks for chest diseases detection. Journal of healthcare engineering. 2018;2018. DOI: 10.1155/2018/4168538.

10. Saul CJ, Urey DY, Taktakoglu CD. Early Diagnosis of Pneumonia with Deep Learning. arXiv preprint arXiv:1904.00937. 2019 Apr 1.

11. Stephen O, Sain M, Maduh UJ, Jeong DU. An Efficient Deep Learning Approach to Pneumonia Classification in Healthcare. Journal of healthcare engineering. 2019;2019. DOI: $10.1155 / 2019 / 4180949$

12. Antin B, Kravitz J, Martayan E. Detecting Pneumonia in Chest X-Rays with Supervised Learning.

13. Saraiva AA, Santos DB, Costa NJ, Sousa JV, Ferreira NF, Valente A, Soares S. Models of Learning to Classify X-ray Images for the Detection of Pneumonia using Neural Networks. 
14. Gu X, Pan L, Liang H, Yang R. Classification of Bacterial and Viral Childhood Pneumonia Using Deep Learning in Chest Radiography. InProceedings of the 3rd International Conference on Multimedia and Image Processing 2018 Mar 16 (pp. 88-93). ACM. DOI: 10.1145/3195588.3195597.

15. Rahman, T., Chowdhury, M.E., Khandakar, A., Islam, K.R., Islam, K.F., Mahbub, Z.B., Kadir, M.A. and Kashem, S., 2020. Transfer Learning with Deep Convolutional Neural Network (CNN) for Pneumonia Detection using Chest X-ray. Applied Sciences, 10(9), p.3233. DOI: 10.3390/app10093233.

16. Rajaraman, S., Candemir, S., Kim, I., Thoma, G. and Antani, S., 2018. Visualization and interpretation of convolutional neural network predictions in detecting pneumonia in pediatric chest radiographs. Applied Sciences, 8(10), p.1715. DOI: 10.3390/app8101715.

17. Kermany DS, Goldbaum M, Cai W, Valentim CC, Liang H, Baxter SL, McKeown A, Yang G, Wu X, Yan F, Dong J. Identifying medical diagnoses and treatable diseases by image-based deep learning. Cell. 2018 Feb 22;172(5):1122-31. DOI: 10.1016/j.cell.2018.02.010.

18. Alqudah AM. AOCT-NET: a convolutional network automated classification of multiclass retinal diseases using spectral-domain optical coherence tomography images. Medical \& Biological Engineering \& Computing. 2019 Nov 14:1-3. DOI: 10.1007/s11517-019-02066-y.

19. Trosten DJ, Sharma P. Unsupervised Feature Extraction-A CNN-Based Approach. InScandinavian Conference on Image Analysis 2019 Jun 11 (pp. 197-208). Springer, Cham. DOI: 10.1007/978-3-03020205-7_17.

20. Narayanan, B.N., De Silva, M.S., Hardie, R.C., Kueterman, N.K. and Ali, R., 2019. Understanding Deep Neural Network Predictions for Medical Imaging Applications. arXiv preprint arXiv:1912.09621.

21. Garcia-Gasulla D, Parés F, Vilalta A, Moreno J, Ayguadé E, Labarta J, Cortés U, Suzumura T. On the behavior of convolutional nets for feature extraction. Journal of Artificial Intelligence Research. 2018 Mar 20;61:563-92. DOI: 10.1613/jair.5756.

22. Alqudah AM. Ovarian Cancer Classification Using Serum Proteomic Profiling and Wavelet Features A Comparison of Machine Learning and Features Selection Algorithms. Journal of Clinical Engineering. 2019 Oct 1;44(4):165-73. DOI: 10.1097/JCE.0000000000000359.

23. Alqudah AM, Algharib HM, Algharib AM, Algharib HM. Computer aided diagnosis system for automatic two stages classification of breast mass in digital mammogram images. Biomedical Engineering: Applications, Basis and Communications. 2019 Feb 10;31(01):1950007. DOI: 10.4015/S1016237219500078.

24. Alquran $\mathrm{H}$, Qasmieh IA, Alqudah AM, Alhammouri S, Alawneh E, Abughazaleh A, Hasayen $F$. The melanoma skin cancer detection and classification using support vector machine. In2017 IEEE Jordan Conference on Applied Electrical Engineering and Computing Technologies (AEECT) 2017 Oct 11 (pp. 1-5). IEEE. DOI: 10.1109/AEECT.2017.8257738

25. Alqudah A, Alqudah AM. Sliding Window Based Support Vector Machine System for Classification of Breast Cancer Using Histopathological Microscopic Images. IETE Journal of Research. 2019 Mar 7:19. DOI: 10.1080/03772063.2019.1583610. 
26. Alqudah AM. Towards classifying non-segmented heart sound records using instantaneous frequency based features. Journal of medical engineering \& technology. 2019 Oct 3;43(7):418-30.

27. Alqudah AM, Alquraan $\mathrm{H}$, Abu-Qasmieh I, Al-Badarneh A. Employing Image Processing Techniques and Artificial Intelligence for Automated Eye Diagnosis Using Digital Eye Fundus Images. In Journal of Biomimetics, Biomaterials and Biomedical Engineering 2018 (Vol. 39, pp. 40-56). Trans Tech Publications. DOI: 10.4028/www.scientific.net/JBBBE.39.40.

28. Alquran H, Alqudah AM, Abu-Qasmieh I, Al-Badarneh A, Almashaqbeh S. ECG CLASSIFICATION USING HIGHER ORDER SPECTRAL ESTIMATION AND DEEP LEARNING TECHNIQUES. Neural Network World. 2019 Jul 1;29(4):207-19. DOI: 10.14311/nnw.2019.29.014.

\section{Figures}

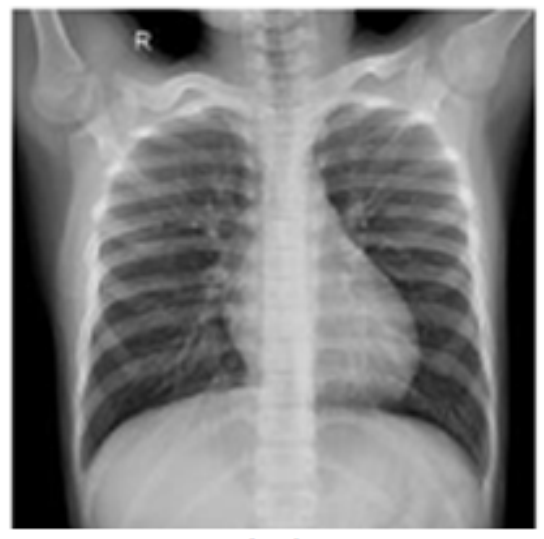

(A)

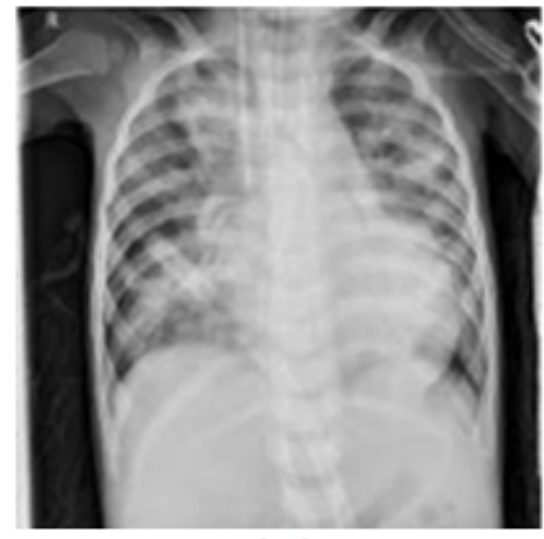

(B)

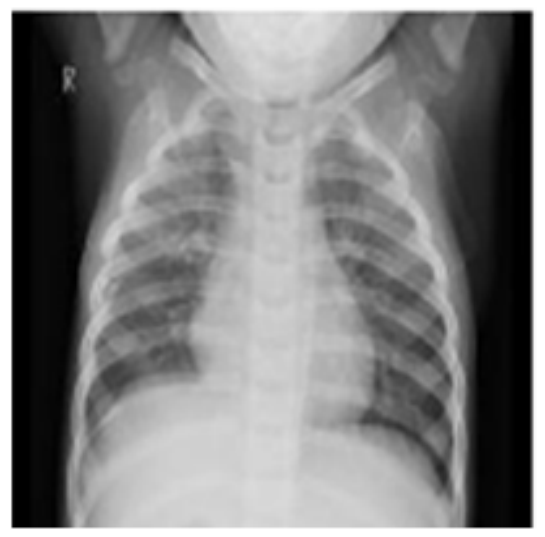

(C)

\section{Figure 1}

Chest x-ray images; A: Normal, B: Bacterial Pneumonia, and C: Viral Pneumonia.
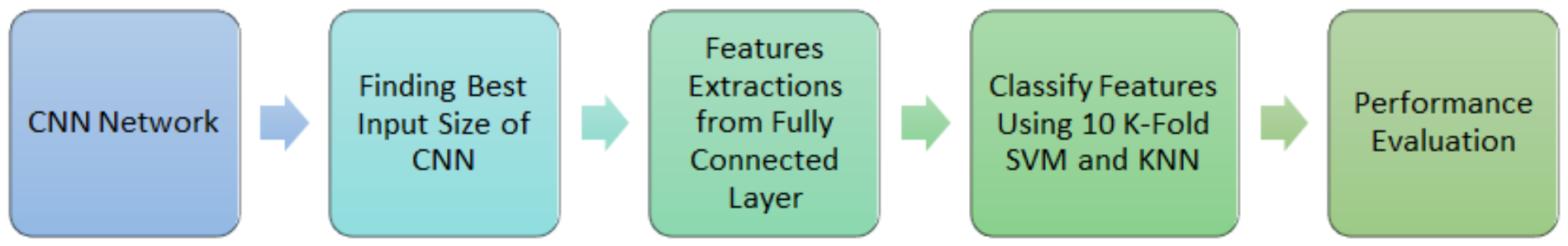

\section{Figure 2}

Block Diagram of the Proposed Methodology. 


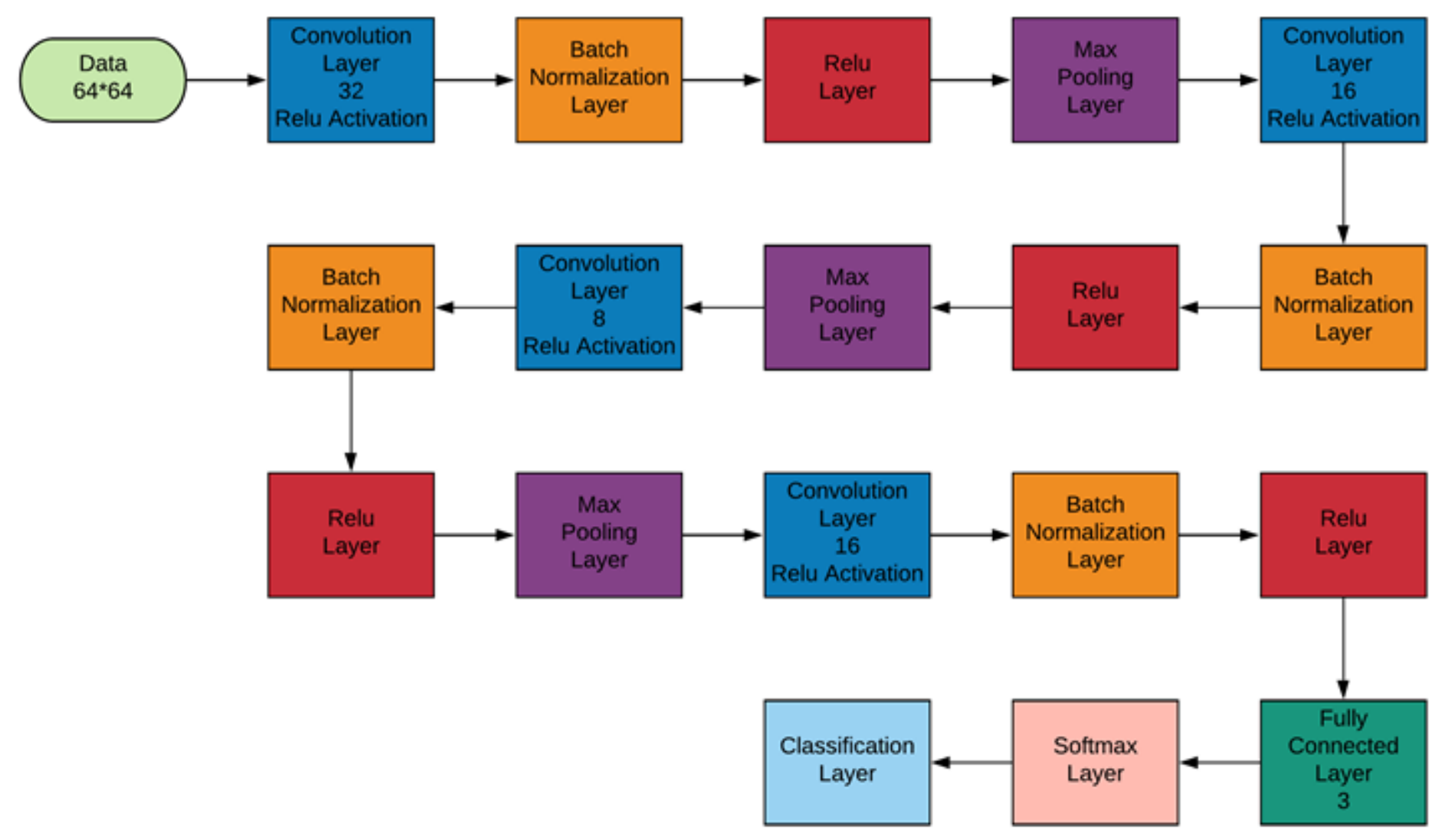

\section{Figure 3}

The Architecture of the Used CNN Model 

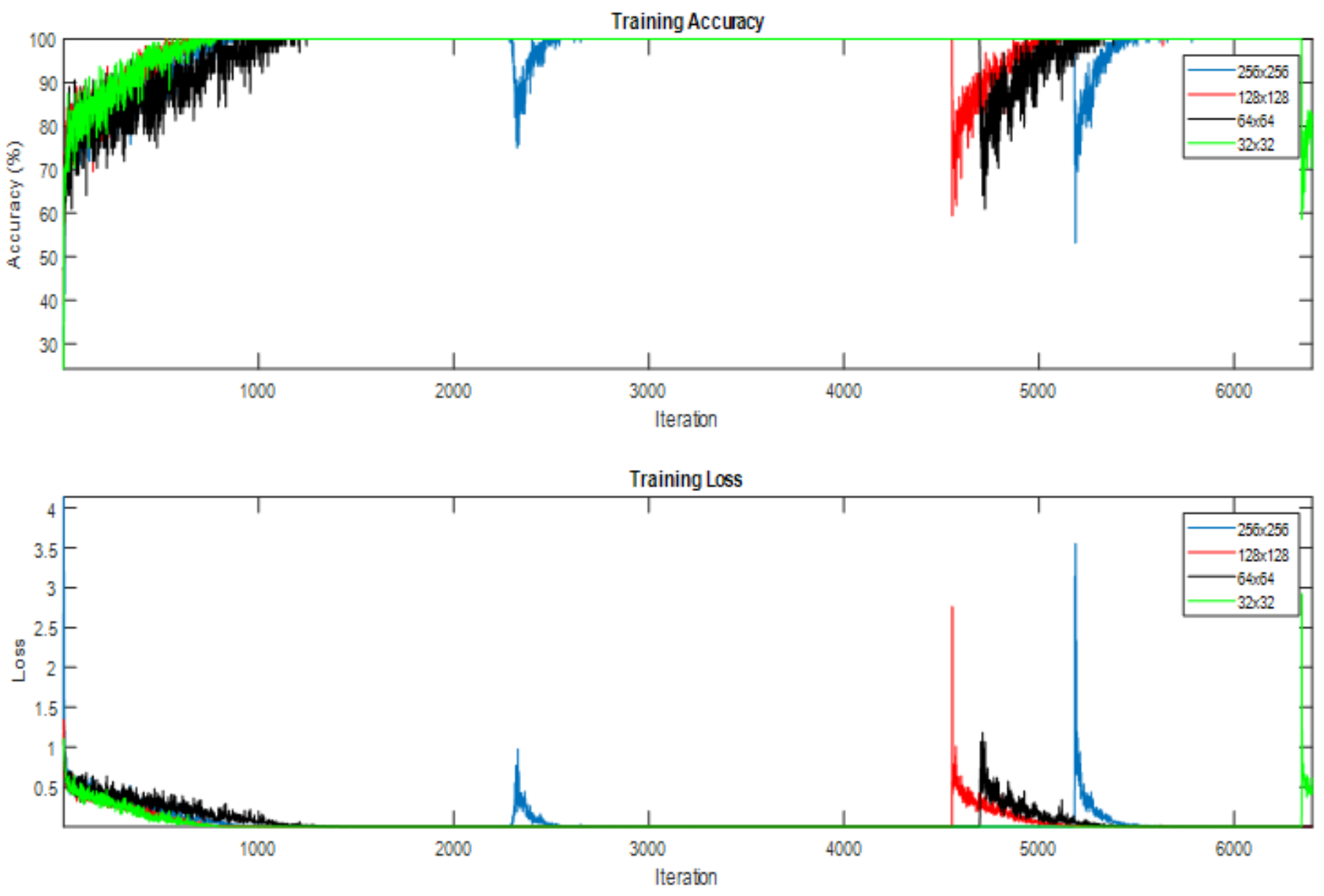

Figure 4

Training Accuracy and Loss for Different Input Sizes 
Original Class: NORMAL

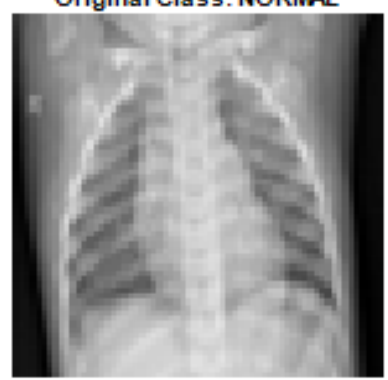

Original Class: PNEUMONIA BACTERIA

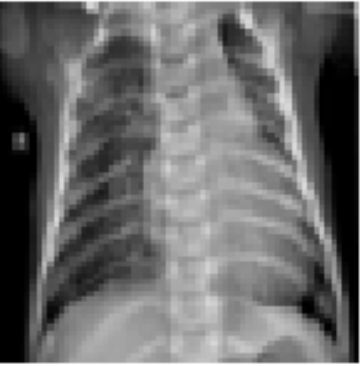

Original Class: PNEUMONIA VIRUS

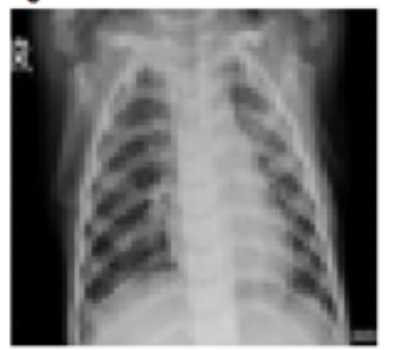

NORMAL, 1

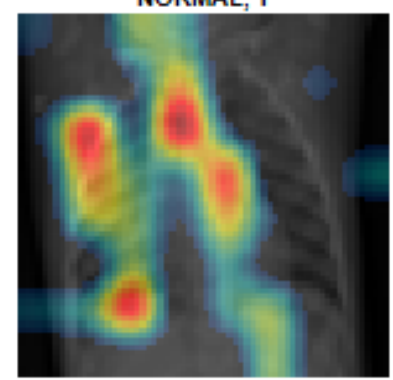

NORMAL, 1.8631e-09

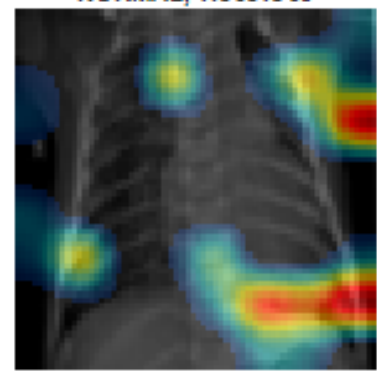

NORMAL, 1.0697e-05

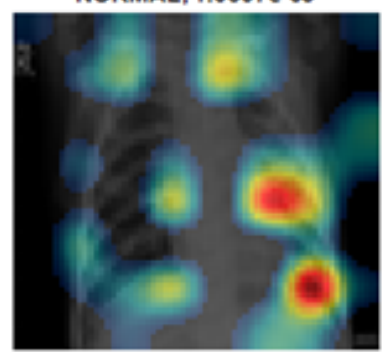

PNEUMONIA BACTERIA, 4.517e-07

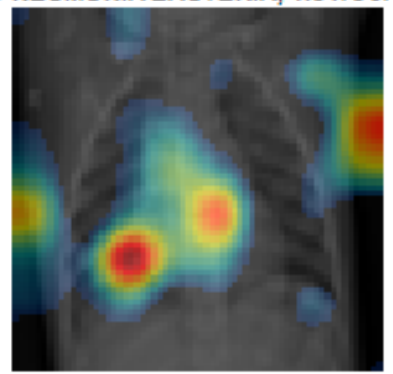

PNEUMONIA BACTERIA, 1

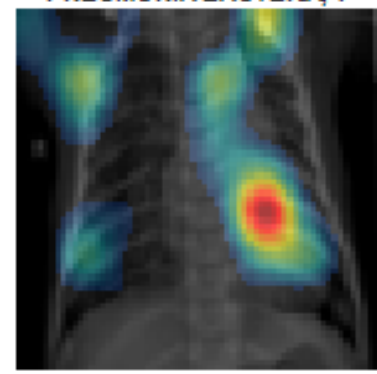

PNEUMONIA BACTERIA, 0.038104

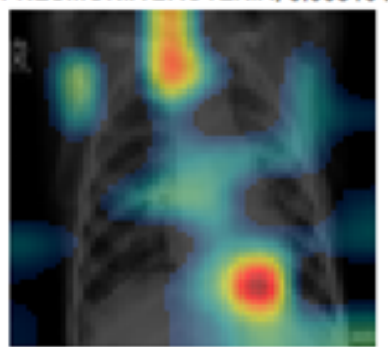

PNEUMONIA VIRUS, $1.0409 \mathrm{e}-08$

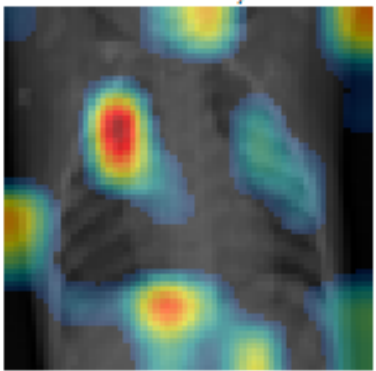

PNEUMONIA VIRUS, 2.3283e-09

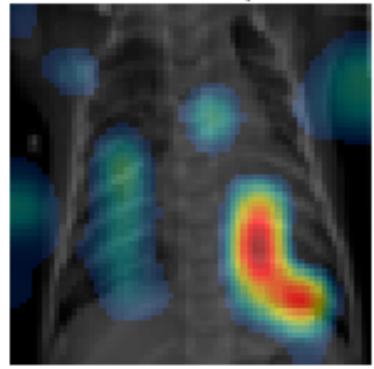

PNEUMONIA VIRUS, 0.96189

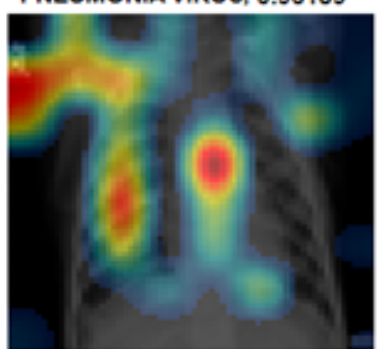

Figure 5

The Class Activation Mapping (CAM) for Three Cases (Normal, Bacterial Pneumonia, and Viral Pneumonia) using Last ReLU Layer. 


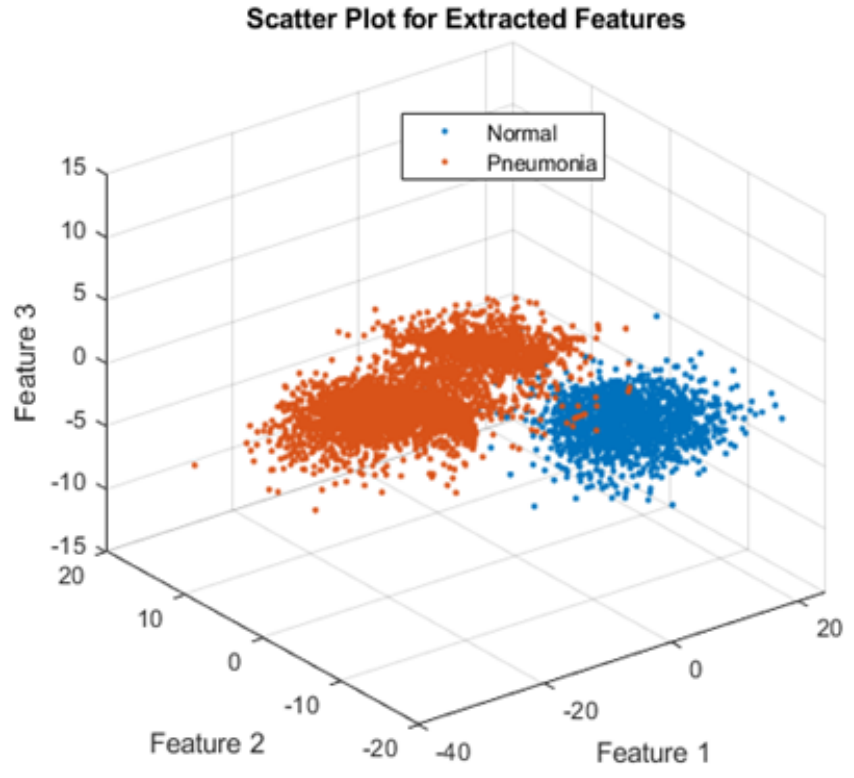

(A)

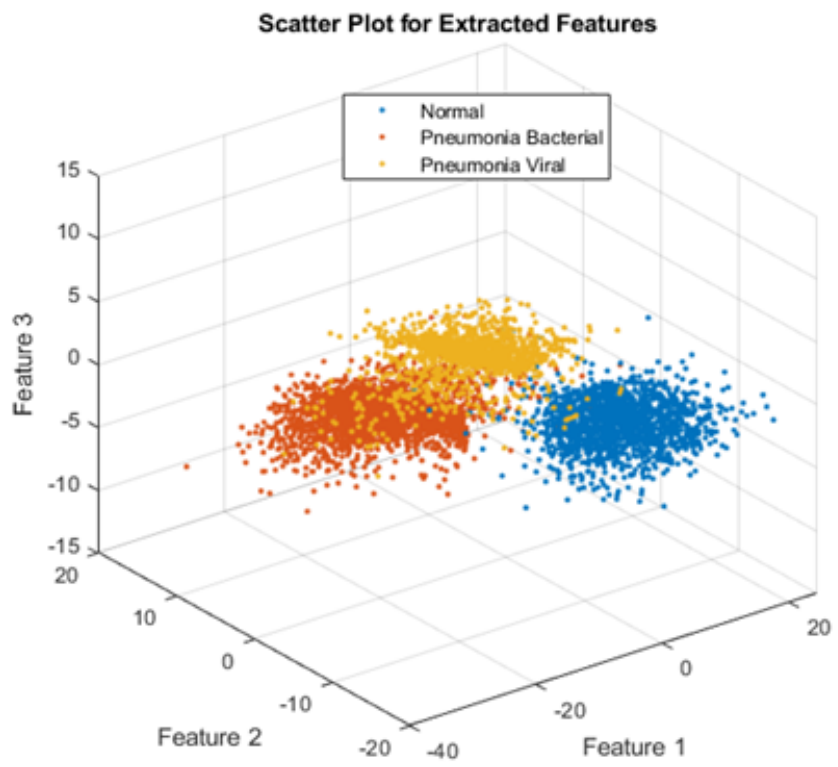

(B)

\section{Figure 6}

The Extracted Features Using the CNN Model; (A) for Two Classes, and (B) for Three Classes.

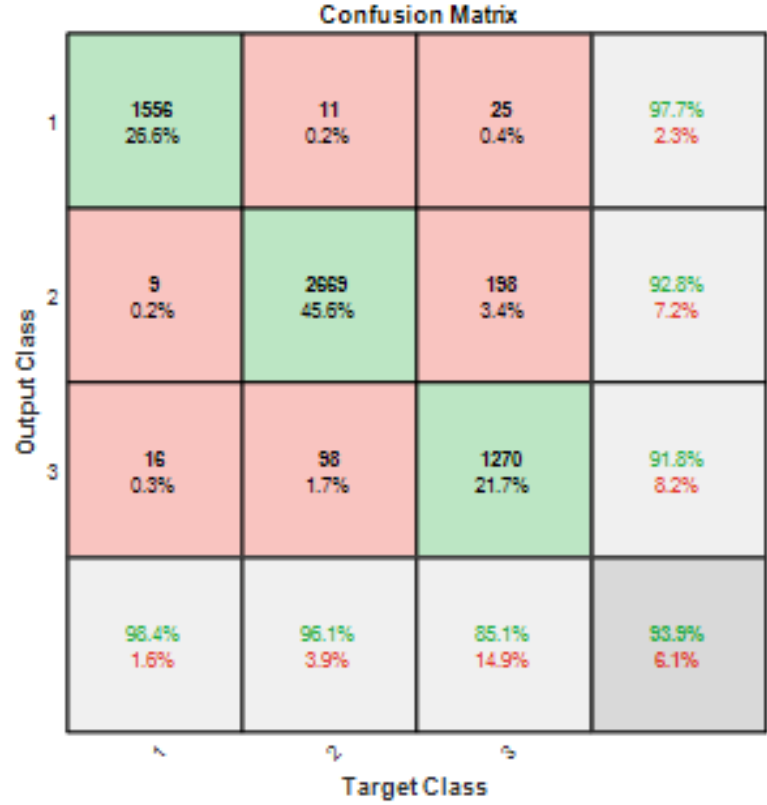

(A)

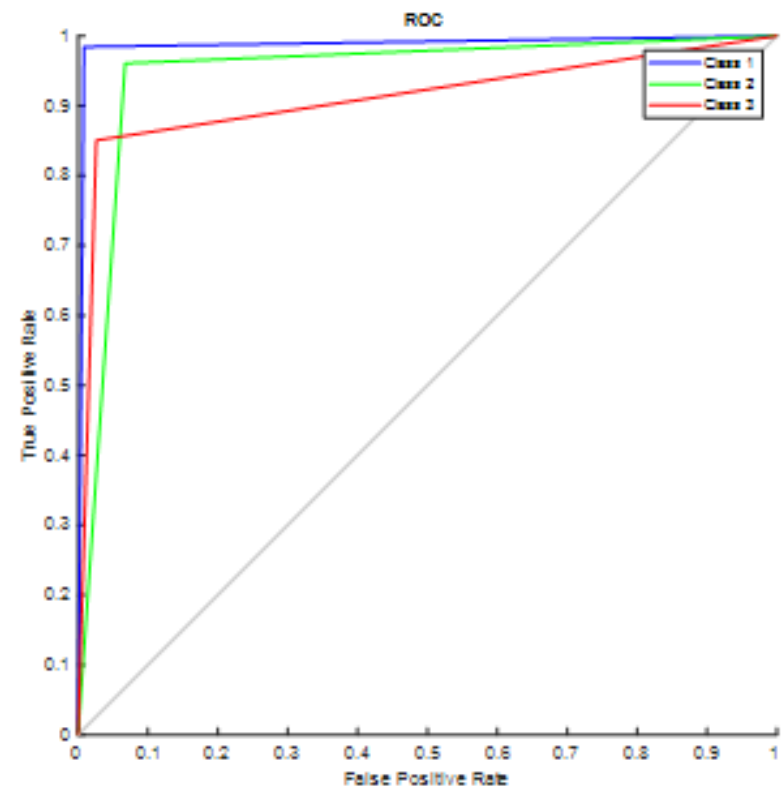

(B)

Figure 7 
The Results of KNN Classifier; (A) Confusion Matrix of the classifier, and (B) ROC of the classifier.

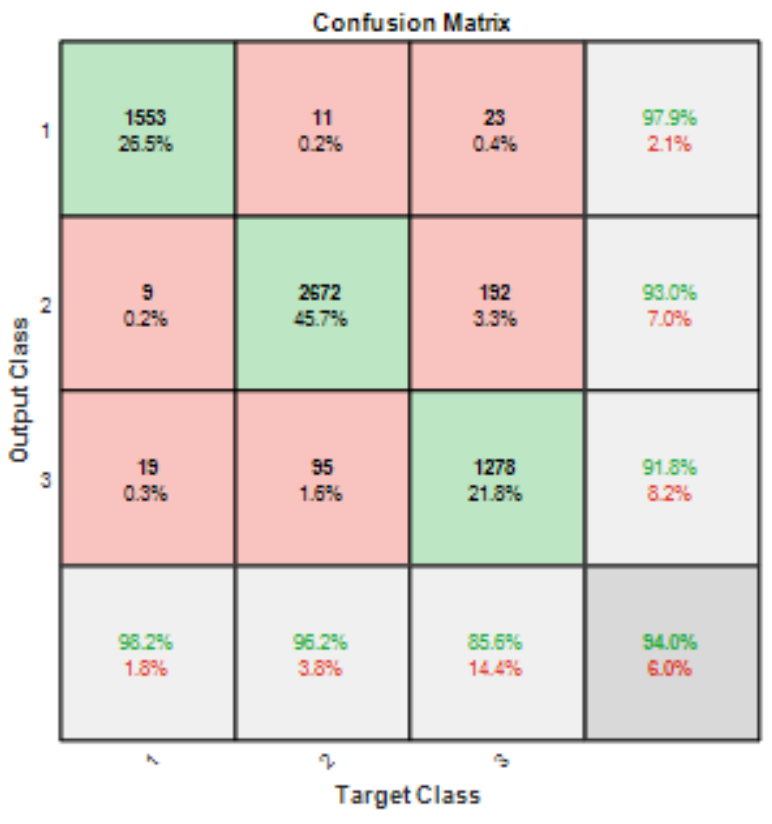

(A)

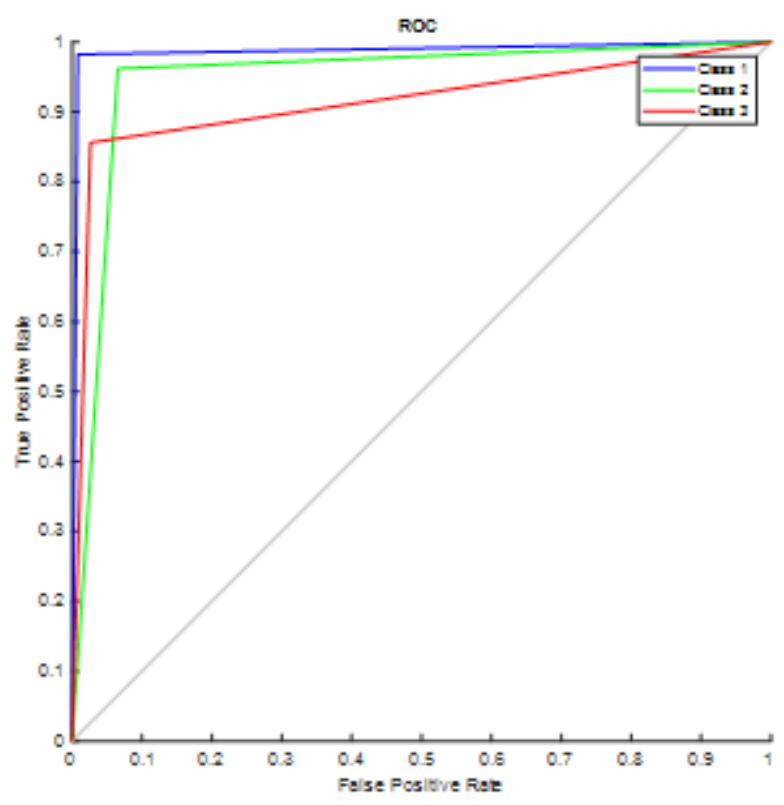

(B)

\section{Figure 8}

The Results of SVM Classifier; (A) Confusion Matrix of the classifier, and (B) ROC of the classifier. 